\title{
Secondary systemic amyloidosis in inflammatory bowel disease: a nationwide analysis
}

\section{Prabin Sharma ${ }^{a}$, Rodrigo Aguilarb, Omer Asif Siddiquic, Mark Abi Nader ${ }^{\mathrm{d}}$}

Yale New Haven Health-Bridgeport Hospital; Marshall University Joan C. Edwards School of Medicine, Huntington; University of Tennessee Health Science Center, Memphis; MedStar Georgetown University Hospital, Washington, USA

\section{Abstract}

Departments of anternal Medicine, Yale New Haven Health-Bridgeport

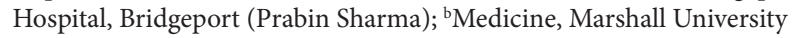
Joan C. Edwards School of Medicine, Huntington (Rodrigo Aguilar); ${ }^{c}$ Medicine, University of Tennessee Health Science Center, Memphis (Omer Asif Siddiqui); ${ }^{\mathrm{d} D i v i s i o n}$ of Nephrology and Hypertension, MedStar Georgetown University Hospital, Washington (Mark Abi Nader), USA

Conflict of Interest: None

Correspondence to: Prabin Sharma, MD, Department of Internal Medicine, Yale New Haven Health-Bridgeport Hospital, 267 Grant Street, Bridgeport, CT 06610, USA, Tel.: +1 203362 7948, Fax: +1 203 384 3135, e-mail: prabin.sharma@bpthosp.org

Received 17 March 2017; accepted 29 May 2017; published online 14 June 2017

DOI: https://doi.org/10.20524/aog.2017.0168

\section{Introduction}

Amyloidosis refers to the deposition of fibrils composed of low-molecular-weight subunits of different plasma proteins in the extracellular tissue $[1,2]$. The two major types of amyloidosis are primary or AL (light-chain) amyloidosis, and secondary or AA amyloidosis [2]. AL amyloidosis, the more common variant, is due to the deposition of immunoglobulin light-chain protein fragments, often associated with plasma cell dyscrasia, such as multiple myeloma, monoclonal gammopathy of undetermined significance, or Waldenström's macroglobulinemia [2,3]. Secondary systemic amyloidosis (SSA) is caused by the deposition of amyloid fibrils derived from circulating acute-phase reactant serum amyloid A protein [4-6]. It is more commonly seen in association with 
chronic conditions, such as inflammatory bowel disease (IBD), rheumatoid arthritis, juvenile rheumatoid arthritis, ankylosing spondylitis, bronchiectasis, Sjögren's syndrome, systemic lupus erythematosus, tuberculosis, Reiter's syndrome, and hereditary fever syndromes such as familial mediterranean fever $[5,6]$. AA amyloidosis is about six times less common than $\mathrm{AL}$ amyloidosis, though it is possible that it may be underdiagnosed [5].

IBD is a chronic inflammatory condition that comprises two disorders, Crohn's disease (CD) and Ulcerative colitis (UC). In the United States, 1.4 million people suffer from these diseases, as do 2.2 million in Europe [7]. The incidence and prevalence of IBD are increasing with time in different parts of the world [8]. IBD is associated with various extra-intestinal manifestations, of which at least one may be present in up to $36 \%$ IBD patients. SSA is a rare extra-intestinal manifestation of this disease [9]. An association between IBD and AA amyloidosis has been reported in prior case reports, case series and observational studies [10-12]. In this study, we aimed to understand the natural history of SSA, including demographics, comorbidity, in-hospital mortality and predictors of mortality in IBD patients.

\section{Patients and methods}

\section{Data source}

We obtained data from the National Inpatient Sample (NIS) hospital discharge database for the period January 1, 2004, through December 31, 2012, from the Healthcare Cost and Utilization Project (HCUP) of the Agency for Healthcare Research and Quality, Rockville, Maryland. The NIS is the largest all-payer inpatient care database in the United States and contains approximately 8 million hospital stays from about 1000 hospitals sampled to approximate a $20 \%$ stratified sample of the US population. It is a component of the Healthcare Cost and Utilization Project (HCUP), sponsored by the Agency for Healthcare Research and Quality. The NIS represents a sample of non-federal, acute-care hospitals in the US and is stratified with respect to hospital ownership/control, bed size, teaching status, location, and region. Each record represents a single patient discharge and contains demographic information, up to 25 diagnoses and 15 procedures, admission type, patient disposition, length of stay, hospital charges, and hospital characteristics [13].

\section{Study sample}

We extracted data based on the International Classification of Diseases, Ninth Revision, Clinical Modification (ICD-9-CM) codes, creating an initial cohort that identified all patients with a primary or secondary discharge diagnosis of IBD (ICD-9-CM 555.0, 555.1, 555.2, 555.9, 556.0, 556.1, $556.2,556.3,556.4,556.5,556.6,556.8,556.9)$ and SSA (ICD-
9-CM 277.39). We excluded other causes of AA and AL amyloidosis in order to rule out confounding factors. Excluded cases, with their respective ICD-9 codes, included those with hemodialysis (39.95), multiple myeloma (203.0), rheumatoid arthritis (714.0), familial mediterranean fever (277.31), ankylosing spondylitis (720.0), bronchiectasis (494.0), cystic fibrosis (277.0), hairy-cell leukemia (202.4), Hodgkin disease (201.9), juvenile rheumatoid arthritis (714.3), Reiter's syndrome (099.3), Sjögren's syndrome (710.2), systemic lupus erythematous (710.0) and tuberculosis (011.9).

\section{Assembly of propensity-matched cohort and statistical analysis}

We calculated a propensity score for each patient, using a stepwise multivariable logistic regression model to detect linearity. In the model, SSA was a dependent variable and baseline demographics and comorbid characteristics were covariates. Using a greedy matching algorithm, we matched UC patients without SSA with UC patients with SSA in a 2:1 ratio. The same matching technique was applied to match CD patients without SSA with CD patients with SSA. Covariates used for propensity matching included baseline demographics, such as age, sex, race, insurance, and smoking status, and comorbidities, such as diabetes, hypertension, hyperlipidemia, hypothyroidism, congestive heart failure, atrial fibrillation, valvular heart disease, cardiomyopathy, coronary artery disease, stroke, obesity, chronic obstructive pulmonary disease, coagulopathy, deep vein thrombosis, chronic liver disease, anemia, bladder cancer, recto-anal cancer, acute tubular necrosis (ATN), chronic kidney disease stages I to V, end stage renal disease (ESRD), Clostridium difficile infection (CDI), sepsis, severe sepsis, bowel surgeries (small and large bowel resection), pyoderma gangrenosum, pancreatitis, malabsorption and toxic megacolon. The NIS provides up to 25 diagnoses and 15 procedural diagnoses associated with the index admission. We used NIS variables to identify patients' age, sex, race and primary payer, and we used ICD-9-CM codes to identify the various comorbidities and procedures. Pearson's $\chi^{2}$ test was used for analysis of hospitalization trends. All P-values were based on 2-sided tests, and were considered statistically significant if $\mathrm{P}<0.05$. All categorical variables were compared using Pearson's $\chi^{2}$ test and continuous variables were analyzed using the paired t-test. Analyses were performed using SAS version 9.3 (SAS Institute, Cary, North Carolina).

\section{Results}

\section{IBD cohort}

Among 71,208,001 patients hospitalized between 2004 and 2012, 500,046 (0.7\%) patients had IBD (UC or CD). Using the exclusion criteria, a cohort of 476,605 patients with IBD 
was included in the final analysis, of which 302,548 (63.5\%) patients had CD, and 174,057 (36.5\%) had UC (Fig. 1).

\section{Prevalence of SSA in CD and UC}

Among the patients with $\mathrm{CD}$ and patients with UC admitted to the hospital between 2004 and 2012, we identified $47(0.02 \%)$ and $36(0.02 \%)$ cases of SSA, respectively.

\section{Annual hospitalization trends of SSA in CD and UC}

In both the group of patients with $\mathrm{CD}+\mathrm{SSA}(\mathrm{P}=0.01)$ and those with UC+SSA $(\mathrm{P}=0.001)$, we noted significantly increasing trends of hospitalization. We found that the annual hospitalization rates for UC and CD patients without SSA also rose significantly during the study period (Table 1 ).

\section{Patient characteristics and comorbidities}

Baseline characteristics, comorbidities and complications are listed and compared between IBD patients with and without SSA in Tables 2 and 3.

\section{Mortality difference of SSA in CD and UC}

In-hospital mortality was significantly higher in the UC+SSA group $(16.7 \%$ vs. $2.1 \%, \mathrm{P}<0.0001)$ before propensity matching, but was not significantly different ( $17.1 \%$ vs. $7.1 \%, \mathrm{P}=0.11$ ) after matching. Likewise, in $\mathrm{CD}$ patients, in-hospital mortality was higher in the amyloidosis group before matching $(6.4 \%$ vs. $1.0 \%, \mathrm{P}=0.0001)$, but this difference was not seen after matching $(6.8 \%$ vs. $2.3 \%$, $\mathrm{P}=0.20$ ) (Table 4).

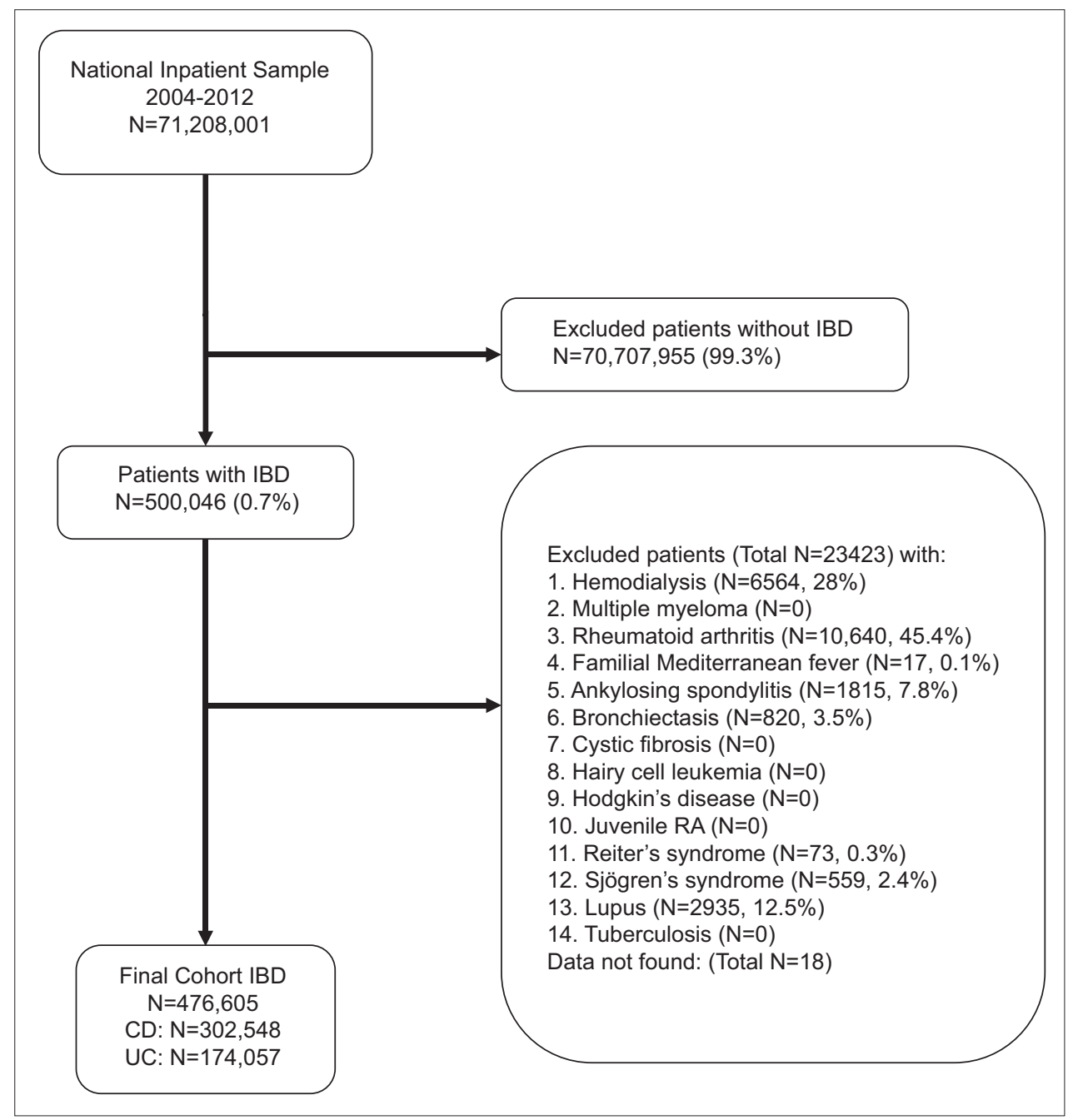

Figure 1 Cohort assembly of patients with diagnosis of CD-UC from 2004 to 2012 in NIS

$I B D$, inflammatory bowel disease; $C D$, Crohn's disease, UC, ulcerative colitis; RA, rheumatoid arthritis 


\section{Predictors of mortality among CD and UC patients with SSA}

Fig. 2A \& B highlight various baseline characteristics and comorbidities that predict mortality in IBD patients with SSA.

Table 1 Annual hospitalization trends of CD+SSA vs. CD-SSA and UC+SSA vs. UC-SSA

\begin{tabular}{|c|c|c|c|c|c|}
\hline \multirow[t]{2}{*}{ Year } & \multicolumn{2}{|c|}{${ }^{\star} \mathrm{CD}+\mathrm{SSA}$} & \multicolumn{2}{|c|}{${ }^{*} \mathrm{CD}-\mathrm{SSA}$} & \multirow[t]{2}{*}{ P-value } \\
\hline & $\mathrm{N}$ & $\%$ & $\mathrm{~N}$ & $\%$ & \\
\hline 2004 & 0 & 0.0 & 28231 & 9.3 & 0.01 \\
\hline 2005 & 0 & 0.0 & 28835 & 9.5 & \\
\hline 2006 & 2 & 4.3 & 28785 & 9.5 & \\
\hline 2007 & 5 & 10.6 & 30321 & 10.0 & \\
\hline 2008 & 10 & 21.3 & 36702 & 12.1 & \\
\hline 2009 & 5 & 10.6 & 35451 & 11.7 & \\
\hline 2010 & 11 & 23.4 & 36814 & 12.1 & \\
\hline 2011 & 5 & 10.6 & 40365 & 13.3 & \\
\hline 2012 & 9 & 19.2 & 36997 & 12.2 & \\
\hline \multirow[t]{2}{*}{ Year } & \multicolumn{2}{|c|}{${ }^{*} \mathrm{UC}+\mathrm{SSA}$} & \multicolumn{2}{|c|}{${ }^{\star} \mathrm{UC}-\mathrm{SSA}$} & $\mathrm{P}$-value \\
\hline & $\mathrm{N}$ & $\%$ & $\mathrm{~N}$ & $\%$ & \\
\hline 2004 & 0 & 0.0 & 16361 & 9.4 & 0.001 \\
\hline 2005 & 0 & 0.0 & 16267 & 9.3 & \\
\hline 2006 & 1 & 2.8 & 16525 & 9.5 & \\
\hline 2007 & 2 & 5.6 & 17689 & 10.2 & \\
\hline 2008 & 3 & 8.3 & 21432 & 12.3 & \\
\hline 2009 & 4 & 11.1 & 20237 & 11.6 & \\
\hline 2010 & 5 & 13.9 & 21252 & 12.2 & \\
\hline 2011 & 11 & 30.6 & 23443 & 13.5 & \\
\hline 2012 & 10 & 27.8 & 20851 & 12.0 & \\
\hline
\end{tabular}

Percentages (\%) are of the total number of patients in each category during the study period

$C D+S S A$, Crohn's disease with secondary systemic amyloidosis; CD-SSA, Crohn's disease without secondary systemic amyloidosis; UC+SSA, ulcerative colitis with secondary systemic amyloidosis; UC-SSA, ulcerative colitis without secondary systemic amyloidosis

\section{Discussion}

Information regarding the epidemiology of SSA is scarce and very few studies have looked at its prevalence specifically in IBD patients. SSA, or AA amyloidosis, has been reported as a complication of IBD and was first described in 1936 [14]. Further research has supported this association between the two conditions [10-12,14-16] and it has been reported that SSA is more frequently present in patients with CD than in those with UC [14]. The incidence of SSA associated with IBD in the United States is estimated at $0.9 \%$ in patients with CD and $0.07 \%$ in those with UC [10]. It has been hypothesized that the predominance of SSA in CD patients may be related to the more pronounced acute phase and a higher prevalence of suppurative features among these patients. Another study suggested that the incidence of amyloidosis secondary to CD is decreasing, probably in response to better and newer treatment methods including the use of biologic agents $[12,17]$.

Our study is the largest so far to evaluate this association and has the highest number of SSA cases in IBD. It highlights for the first time that the prevalence of SSA in hospitalized patients with IBD is much lower than previously reported and is the same in patients with $\mathrm{CD}(0.02 \%)$ and those with UC $(0.02 \%)$. Moreover, we highlight an increase in the hospitalization rates of IBD patients in general, as well as IBD patients with SSA, over a 9-year period. The implication of this rise in hospitalizations is unclear, but it may be related to an improved awareness, detection and newer treatment methods of the disease, as well as to the increased life expectancy of these patients.

This study also showcases the mortality burden afflicted by SSA on IBD patients. The main predictors of mortality of hospitalized IBD patients with SSA compared to the control group are older age, severe sepsis, ESRD, ATN, coagulopathy, colon ischemia, recto-anal cancer, CDI and small bowel resection. An awareness of these complications and efforts to prevent them may help improve the prognosis of these patients. Though we noted that SSA does not influence inhospital mortality in IBD patients, it is likely that SSA plays a role in their long-term outcomes. Studies including patients with outpatient follow up and those looking at readmissions will help further elucidate the prognosis of SSA.
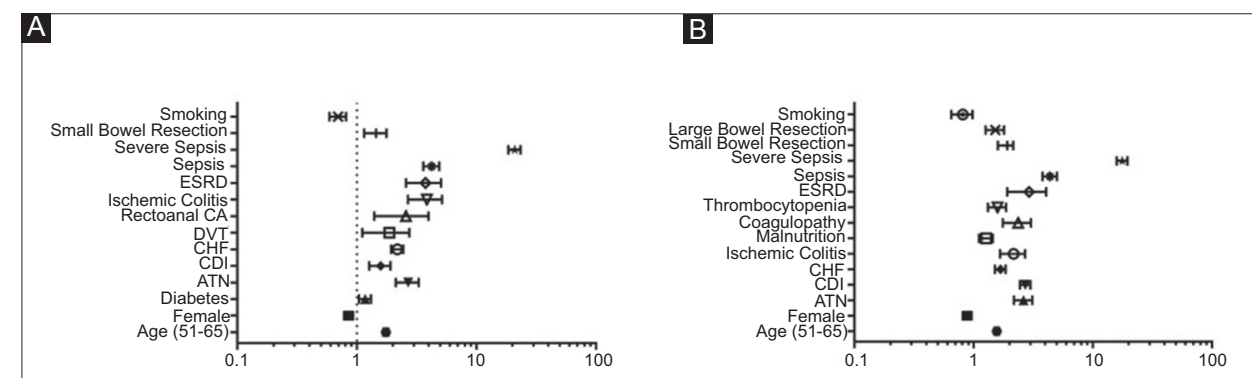

Figure 2 (A) Mortality predictors of Crohn's disease patients with secondary systemic amyloidosis (CD+SSA). (B) Mortality predictors of ulcerative colitis patients with secondary systemic amyloidosis (UC+SSA)

ATN, acute tubular necrosis; CHF, congestive heart failure; DVT, deep-vein thrombosis; CA, cancer; ESRD, end-stage renal disease; CDI, Clostridium difficile 
Table 2 Baseline clinical characteristics of all the patients (before and after propensity matching) CD+SSA vs. CD-SSA

\begin{tabular}{|c|c|c|c|c|c|c|}
\hline \multirow[t]{4}{*}{ Variables } & \multicolumn{3}{|c|}{ Full Cohort } & \multicolumn{3}{|c|}{ Matched Cohort } \\
\hline & $\mathrm{CD}+\mathrm{SSA}$ & CD-SSA & $\mathrm{P}$-value & $\mathrm{CD}+\mathrm{SSA}$ & CD-SSA & P-value \\
\hline & N (\%) & N (\%) & & N (\%) & N (\%) & \\
\hline & $\mathrm{N}=47$ & $\mathrm{~N}=302548$ & & $\mathrm{~N}=44$ & $\mathrm{~N}=88$ & \\
\hline Age (years) & & & $<0.001$ & & & 0.16 \\
\hline$<21$ & $3(6.4)$ & $16093(5.8)$ & & $3(6.8)$ & $5(5.8)$ & \\
\hline $22-35$ & 0 & $61947(22.2)$ & & 0 & $11(12.8)$ & \\
\hline $36-50$ & $6(12.8)$ & $71930(25.8)$ & & $6(11.4)$ & $15(17.5)$ & \\
\hline $51-65$ & $19(40.4)$ & $66820(23.9)$ & & $18(40.9)$ & $25(29.1)$ & \\
\hline $66-80$ & $14(29.8)$ & $44520(15.9)$ & & $14(31.8)$ & $23(26.7)$ & \\
\hline$>81$ & $5(10.6)$ & $17757(6.4)$ & & $4(9.1)$ & $7(8.1)$ & \\
\hline Sex & & & 0.44 & & & 0.80 \\
\hline Female & $25(53.2)$ & $177612(58.8)$ & & $24(54.5)$ & $46(52.3)$ & \\
\hline Male & $22(46.8)$ & $124510(41.2)$ & & $20(45.5)$ & $42(47.7)$ & \\
\hline Race & & & 0.83 & & & 0.52 \\
\hline Caucasian & $36(90)$ & $201660(82.2)$ & & $33(89.2)$ & $64(91.4)$ & \\
\hline African American & $3(7.5)$ & 25598 (10.4) & & $3(8.1)$ & $2(2.9)$ & \\
\hline Hispanic & $1(2.5)$ & $10206(4.2)$ & & $1(2.7)$ & $1(1.4)$ & \\
\hline Asian & 0 & $1796(0.7)$ & & 0 & $2(2.9)$ & \\
\hline Native American & 0 & $860(0.4)$ & & 0 & 0 & \\
\hline Other & 0 & $5133(2.1)$ & & 0 & $1(1.4)$ & \\
\hline Primary payer & & & 0.03 & & & 0.56 \\
\hline Medicare & $25(53.2)$ & $99706(33.0)$ & & $23(52.3)$ & $41(46.6)$ & \\
\hline Medicaid & 0 & 37992 (12.6) & & 0 & $3(3.4)$ & \\
\hline Private & $18(38.3)$ & $136184(45.1)$ & & 17 (38.6) & $40(45.5)$ & \\
\hline Self pay & $3(6.4)$ & $16224(5.4)$ & & $3(6.8)$ & $3(3.4)$ & \\
\hline No charge & 0 & $1777(0.6)$ & & 0 & 0 & \\
\hline Other & $1(2.1)$ & $9940(3.3)$ & & $1(2.3)$ & $1(1.1)$ & \\
\hline ATN & $3(6.4)$ & $1448(0.5)$ & $<0.0001$ & $3(6.8)$ & $7(7.9)$ & 0.81 \\
\hline $\mathrm{CHF}$ & $10(21.3)$ & $15370(5.1)$ & $<0.0001$ & $8(18.2)$ & $18(20.5)$ & 0.76 \\
\hline Atrial fibrillation & 7 (14.9) & $15947(5.3)$ & 0.01 & $6(13.6)$ & $12(13.6)$ & 1.00 \\
\hline Cardiomyopathy & $13(27.7)$ & $4141(1.4)$ & $<0.0001$ & $11(25.0)$ & $23(26.1)$ & 0.88 \\
\hline Thrombocytopenia & $5(10.6)$ & $4988(1.7)$ & $<0.0001$ & $5(11.4)$ & $10(11.4)$ & 1.00 \\
\hline Severe sepsis & $3(6.4)$ & $3552(1.2)$ & 0.01 & $3(6.8)$ & $2(2.3)$ & 0.19 \\
\hline ESRD & $4(8.5)$ & $798(0.3)$ & $<0.0001$ & $4(9.1)$ & $8(9.1)$ & 1.00 \\
\hline
\end{tabular}

CD+SSA, Crohn's disease with secondary systemic amyloidosis; CD-SSA, Crohn's disease without secondary systemic amyloidosis; ATN, acute tubular necrosis; CHF, congestive heart failure; ESRD, end-stage renal disease

SSA disease is known to involve multiple organ systems, including cardiac, renal, autonomic and adrenal, among others [5]. We found that IBD patients with SSA tend to have a significantly higher incidence of ATN, with more rapid progression to advanced chronic kidney disease and ESRD. This may point towards the aggressive nature of the renal injury and rapid progression of the disease. Tubular injury in this population can be the result of toxicity from anti-inflammatory medications, subclinical ischemia related to dehydration and hypotension, or the use of intravenous contrast for diagnostic purposes. This further highlights the importance of avoiding nephrotoxic agents in these patients, 
Table 3 Baseline clinical characteristics of total patients (before and after propensity matching score) UC+SSA vs. UC-SSA

\begin{tabular}{|c|c|c|c|c|c|c|}
\hline \multirow[t]{4}{*}{ Variables } & \multicolumn{3}{|c|}{ Full cohort } & \multicolumn{3}{|c|}{ Matched cohort } \\
\hline & $\mathrm{UC}+\mathrm{SSA}$ & UC-SSA & $\mathrm{P}$-value & $\mathrm{UC}+\mathrm{SSA}$ & UC-SSA & P-value \\
\hline & N (\%) & N (\%) & & N (\%) & $\mathrm{N}(\%)$ & \\
\hline & $\mathrm{N}=36$ & $\mathrm{~N}=174057$ & & $\mathrm{~N}=35$ & $\mathrm{~N}=70$ & \\
\hline Age (years) & & & $<0.04$ & & & 0.37 \\
\hline$<21$ & $2(5.6)$ & $9170(5.7)$ & & $2(5.7)$ & $5(7.6)$ & \\
\hline $21-35$ & $1(2.8)$ & $27626(17.3)$ & & $1(2.9)$ & $6(9.1)$ & \\
\hline $36-50$ & $3(8.3)$ & $31948(19.9)$ & & $3(8.6)$ & $7(10.6)$ & \\
\hline $51-65$ & $11(30.6)$ & $36959(23.0)$ & & $11(31.4)$ & $9(13.6)$ & \\
\hline $66-80$ & $13(36.1)$ & $35782(22.3)$ & & $13(34.3)$ & $27(40.9)$ & \\
\hline$>81$ & $6(16.6)$ & $18946(11.8)$ & & $6(17.1)$ & $12(18.2)$ & \\
\hline Sex & & & 0.44 & & & 0.68 \\
\hline Female & $19(52.8)$ & $80436(46.30)$ & & $19(54.3)$ & $35(50.0)$ & \\
\hline Male & $17(47.2)$ & $93299(53.70)$ & & $16(45.7)$ & $35(45.7)$ & \\
\hline Race & & & 0.19 & & & 0.21 \\
\hline Caucasian & $22(71.0)$ & $114728(79.7)$ & & $21(70.0)$ & $53(86.9)$ & \\
\hline African American & $6(19.3)$ & $12297(8.5)$ & & $6(20.0)$ & $5(8.2)$ & \\
\hline Hispanic & $1(3.2)$ & $10482(7.3)$ & & $1(3.3)$ & $2(3.3)$ & \\
\hline Asian & 0 & $1972(1.4)$ & & 0 & 0 & \\
\hline Native American & 0 & $580(0.4)$ & & 0 & 0 & \\
\hline Other & $2(6.5)$ & $3963(2.7)$ & & $2(6.7)$ & $1(37.0)$ & \\
\hline Primary payer & & & 0.09 & & & 0.72 \\
\hline Medicare & $20(55.5)$ & $64316(37.0)$ & & $19(54.3)$ & $44(62.9)$ & \\
\hline Medicaid & $3(8.3)$ & $15531(8.9)$ & & $3(8.5)$ & $5(7.1)$ & \\
\hline Private & $10(27.8)$ & $78992(45.5)$ & & $10(28.5)$ & $17(24.3)$ & \\
\hline Self pay & $1(2.8)$ & $7718(4.4)$ & & $1(2.9)$ & 0 & \\
\hline No charge & $1(2.8)$ & $924(0.5)$ & & $1(2.9)$ & $1(1.4)$ & \\
\hline Other & $1(2.8)$ & $6259(3.6)$ & & $1(2.9)$ & $3(4.3)$ & \\
\hline Hyperlipidemia & $12(33.3)$ & $31663(18.2)$ & 0.02 & $11(31.4)$ & $20(28.6)$ & 0.76 \\
\hline Hypothyroidism & $7(19.4)$ & $15587(9.0)$ & 0.03 & $7(20.0)$ & $11(15.7)$ & 0.58 \\
\hline ATN & $4(11.1)$ & $1390(0.8)$ & $<0.0001$ & $4(11.4)$ & $8(11.4)$ & 1.00 \\
\hline CDI & $5(13.9)$ & $9948(5.7)$ & 0.03 & $5(14.3)$ & $8(11.4)$ & 0.67 \\
\hline CHF & $10(27.8)$ & $12595(7.2)$ & $<0.0001$ & $10(28.6)$ & $19(27.1)$ & 0.88 \\
\hline Atrial Fibrillation & $7(19.4)$ & $14217(8.2)$ & 0.01 & $7(14.3)$ & $10(20.0)$ & 0.45 \\
\hline Cardiomyopathy & $9(25)$ & $2976(1.7)$ & $<0.0001$ & $9(25.7)$ & $18(25.7)$ & 1.00 \\
\hline Severe Sepsis & $3(6.4)$ & $3552(1.2)$ & 0.01 & $3(8.6)$ & $8(11.4)$ & 0.65 \\
\hline CKD III & $5(13.9)$ & $2303(1.3)$ & $<0.0001$ & $4(11.4)$ & $8(11.4)$ & 1.00 \\
\hline ESRD & $6(16.7)$ & $443(0.3)$ & $<0.0001$ & $6(17.1)$ & $12(17.1)$ & 1.00 \\
\hline
\end{tabular}

UC+SSA, ulcerative colitis with secondary systemic amyloidosis; UC-SSA, ulcerative colitis without secondary systemic amyloidosis; ATN, acute tubular necrosis; CDI, Clostridium difficile infection; CHF, congestive heart failure; CKD III, chronic kidney disease stage III; ESRD, end-stage renal disease

avoiding unnecessary intravenous contrast imaging and encouraging adequate hydration.
Cardiac involvement is another important finding of our paper, in contrast with many previous reports $[18,19]$. In a 
Table 4 Mortality rates in CD with SSA and CD without SSA before and after matching and UC with SSA and UC without SSA before and after matching

Variables
Full cohort pre-matching

$\begin{array}{ccc}{ }^{*} \mathrm{CD}+\mathrm{SSA} & { }^{*} \mathrm{CD}-\mathrm{SSA} & \text { P-value } \\ \mathrm{N}(\%) & \mathrm{N}(\%) & \end{array}$

Matched cohort

\begin{tabular}{ccc}
\hline${ }^{*} \mathrm{CD}+\mathrm{SSA}$ & ${ }^{*} \mathrm{CD}-\mathrm{SSA}$ & P-value \\
$\mathrm{N}(\%)$ & $\mathrm{N}(\%)$ & \\
\hline
\end{tabular}

\begin{tabular}{lccc}
\hline Mortality & $3 \pm 6.4$ & $2873 \pm 1.0$ & 0.0001 \\
\hline Variables & \multicolumn{3}{c}{ Full cohort pre-matching } \\
\cline { 2 - 4 } & ${ }^{*} \mathrm{UC+SSA}$ & ${ }^{*} \mathrm{UC}-\mathrm{SSA}$ & P-value \\
& $\mathrm{N}(\%)$ & $\mathrm{N}(\%)$ &
\end{tabular}

$3676 \pm 2.1$

$<0.0001$

$2 \pm 2.3$

0.20

\begin{tabular}{|c|c|c|c|c|c|c|}
\hline Mortality & $6 \pm 16.7$ & $3676 \pm 2.1$ & $<0.0001$ & $6 \pm 17.1$ & $5 \pm 7.1$ & 0.11 \\
\hline
\end{tabular}

without amyloidosis; \pm standard deviation

study by Tanaka et al, cardiac involvement in AA amyloidosis was suggested to be a predictor of a poor outcome among these patients [20]. Likewise, in our analysis, IBD patients with SSA were significantly associated with cardiac comorbidities, including cardiomyopathy, heart failure and atrial fibrillation. Unfortunately, the NIS is unable to provide us with information as to whether cardiac involvement was confirmed by biopsy; thus, we are unable to determine if this higher rate of cardiac diseases is truly from direct amyloid deposition. Nonetheless, our study demonstrates that cardiac disease remains an important complication and adds significantly to these patients' morbidity. This highlights the importance of an early cardiac workup and careful follow up in these patients, with monitoring for these manifestations. Another important finding of our study is that UC patients who developed SSA had high rates of CDI and severe sepsis. This reaffirms the importance of the cautious use of antibiotics, monitoring for CDI symptoms among these patients.

Our study has an observational and retrospective design, which has its limitations. As seen in other studies that used large administrative databases, the NIS database is prone to inadequate coding and coding errors. Our study was only able to analyze in-hospital outcomes during admission and is thus unable to estimate long-term outcomes. There is a possibility that, given the large number of variables used for comparison between the two groups, some of our results may be attributable to mere coincidence. Whether the increases in hospital stay and in-hospital mortality are attributable to consequences of the amyloidosis itself or to the severity of the underlying IBD cannot be determined. Likewise, we were unable to report whether the cases of SSA in the NIS database were confirmed by biopsy. The NIS lacks medical records and information on laboratory results, limiting our ability to investigate changes and outcomes regarding specific therapeutics. We were also unable to determine the best modality of treatment and treatment outcomes in these cases. The NIS database used for this analysis is also unable to recognize readmissions. Given the type of data available from NIS, no identifiable risk factors for the occurrence of SSA in IBD, such as fistulizing disease, disease location (colon vs.

\section{Summary Box}

\section{What is already known:}

- Secondary systemic amyloidosis (SSA) is a rare but severe extra-intestinal manifestation of inflammatory bowel disease (IBD) with an estimated incidence of $0.9 \%$ in patients with Crohn's disease (CD) and $0.07 \%$ in patients with ulcerative colitis (UC) in the United States

- SSA disease may have multi-system involvement, including the renal, cardiac and autonomic systems, among others

- Patients with UC are more likely to develop Clostridium difficile infection (CDI)

\section{What the new findings are:}

- The prevalence of SSA among hospitalized IBD patients is much lower than previously reported and it occurs with equal prevalence in patients with CD (0.02\%) and those with UC (0.02\%)

- Hospitalization rates of IBD patients and IBD patients with SSA have been rising in the past decade

- IBD patients with SSA have a higher degree of multi-system involvement. They are more likely to progress to end-stage renal disease and develop cardiomyopathy

- UC patients with SSA are at further higher risk of developing CDI and severe sepsis

small bowel), disease duration, use of steroids or biologics or other drugs, etc., could be ascertained. Despite our attempts to avoid and minimize confounding factors, there is a possibility that some may still be present. Further studies are required to validate our results. In spite of these limitations, the use of 
propensity matching made our analysis and between-group comparisons more robust.

In conclusion, SSA is a rare form of disease in patients with IBD, equally common in both CD and UC. A delay in the diagnosis of SSA can adversely affect the prognosis of patients with IBD. These patients can have multiple organ involvement, with maximum impact in the kidneys and the heart. Physicians and gastroenterologists involved in the treatment of these patients should be aware of these complications and should be vigilant in identifying early signs and symptoms to prevent disease progression among the patient population at risk. Patients with IBD should thus be routinely screened for proteinuria with urinary dipsticks and serial measurement of serum amyloid A protein concentration. Furthermore, we stress the role of early cardiac workup, adequate hydration, avoidance of nephrotoxic substances and the cautious use of antibiotics in these patients. Treatment of the underlying disease, $\mathrm{UC}$ or $\mathrm{CD}$, and minimizing flare-ups may be sufficient to prevent the further progress of disease and organ damage.

\section{References}

1. Merlini G, Seldin DC, Gertz MA. Amyloidosis: pathogenesis and new therapeutic options. J Clin Oncol 2011;29:1924-1933.

2. Nasr SH, Schwarz R, D'Agati VD, Markowitz GS. Paraplegia, proteinuria, and renal failure. Kidney Int 2006;69:412-415.

3. Sanchorawala V. Light-chain (AL) amyloidosis: diagnosis and treatment. Clin J Am Soc Nephrol 2006;1:1331-1341.

4. Lachmann HJ, Goodman HJ, Gilbertson JA, et al. Natural history and outcome in systemic AA amyloidosis. N Engl J Med 2007;356:2361-2371.

5. Pinney JH, Lachmann HJ. Systemic AA amyloidosis. Subcell Biochem 2012;65:541-564.

6. Obici L, Merlini G. Amyloidosis in autoinflammatory syndromes. Autoimmun Rev 2012;12:14-17.
7. Loftus EV Jr. Clinical epidemiology of inflammatory bowel disease: Incidence, prevalence, and environmental influences. Gastroenterology 2004;126:1504-1517.

8. Molodecky NA, Soon IS, Rabi DM, et al. Increasing incidence and prevalence of the inflammatory bowel diseases with time, based on systematic review. Gastroenterology 2012;142:46-54.

9. Ardizzone S, Puttini PS, Cassinotti A, Porro GB. Extraintestinal manifestations of inflammatory bowel disease. Dig Liver Dis 2008;40(Suppl 2):S253-S259.

10. Greenstein AJ, Sachar DB, Panday AK, et al. Amyloidosis and inflammatory bowel disease. A 50 -year experience with 25 patients. Medicine (Baltimore) 1992;71:261-270.

11. Wester AL, Vatn MH, Fausa O. Secondary amyloidosis in inflammatory bowel disease: a study of 18 patients admitted to Rikshospitalet University Hospital, Oslo, from 1962 to 1998. Inflamm Bowel Dis 2001;7:295-300.

12. Sattianayagam PT, Gillmore JD, Pinney JH, et al. Inflammatory bowel disease and systemic AA amyloidosis. Dig Dis Sci 2013;58:1689-1697.

13. HCUP Nationwide Inpatient Sample (NIS). Healthcare Cost and Utilization Project (HCUP). 2011. Agency for Healthcare Research and Quality, Rockville, MD. Available at: www.hcup-us.ahrq.gov/ nisoverview.jsp.

14. Moschkowitz E. The clinical aspects of amyloidosis. Ann Intern Med 1936;10:73-89.

15. Cohen H, Fishman AP. Regional enteritis and amyloidosis. Gastroenterology 1949;12:502-508.

16. Serra I, Oller B, Mañosa M, et al. Systemic amyloidosis in inflammatory bowel disease: retrospective study on its prevalence, clinical presentation, and outcome. J Crohns Colitis 2010;4:269-274.

17. Weterman IT, Biemond I, Peña AS. Mortality and causes of death in Crohn's disease. Review of 50 years' experience in Leiden University Hospital. Gut 1990;31:1387-1390.

18. Dubrey SW, Cha K, Simms RW, Skinner M, Falk RH. Electrocardiography and doppler echocardiography in secondary (AA) amyloidosis. Am J Cardiol 1996;77:313-315.

19. Falk RH. Diagnosis and management of the cardiac amyloidoses. Circulation 2005;112:2047-2060.

20. Tanaka F, Migita K, Honda S, et al. Clinical outcome and survival of secondary (AA) amyloidosis. Clin Exp Rheumatol 2003;21:343-346. 